\title{
The Aid Triangle: Recognising the Human Dynamics of Dominance, Justice and Identity
}

\author{
Malcolm MacLachlan, Stuart C. Carr and Eilish McAuliffe \\ Zed Books (2010) \\ Reviewed by Biman Prasad, Faculty of Business and Economics, The University of the South Pacific, Suva, Fiji Islands
}

\begin{abstract}
The dynamics of international aid have always been a subject of intense debate, not only among development researchers but also between donors and recipient governments. This new book, The Aid Triangle, provides a new and fresh perspective on the human dimensions and dynamics of aid and how, if managed well, aid could become the real impetus for the development and progress of the poor. The central theme of the book is that aid is about people and its allocation should put people first by giving consideration to what is important for their lives. The aid literature is littered with analyses of how and why aid has not been effective. Much of it is concerned about the processes through which aid is disbursed, how it is evaluated and what is the real motivation of aid donors. More often than not, economists have placed a lot of emphasis on how aid can contribute to economic growth, which is essential for improving quality of life. The Aid Triangle is different in that it cuts across the human dimensions of aid by arguing that dominance, justice and identity are the primary concerns that motivate people and should be central in any analysis of aid effectiveness.
\end{abstract}

\section{Dominance}

The authors carefully use social dominance theory to provide a different perspective on aid and development. There is no doubt that the donors and the workers involved in implementing aid strategies are important players. Many consider their importance and role as superior to that of the aid recipients. Usually, the local counterparts who work with aid workers feel a sense of powerlessness in determining or assessing what might be the best strategies for implementing aid programs. The authors rightly observe that 'the stratification of relations is a key impediment to development'. Those who manage aid on behalf of their countries or international organisations have certainly shown arrogance and sometime condescending attitudes in their assessment of the capacity of local politicians and policymakers to provide advice. Usually, the project proposals provide a rigid framework that does not allow much flexibility for local stakeholders to change things to suit their circumstances. It is often said that the rigidness displayed by those who manage the aid, and the conditions attached to the aid, are necessary to minimise abuse and prevent pilferage. It is true that there is widespread abuse and wastage of aid money. But this situation exists partly because of the unsound allocation, distribution and implementation of aid due to the lack of local input in the decision-making process.

\section{Justice}

This chapter provides an exceptional analysis of the idea of justice and why this should be an important consideration for all those involved in the aid industry. While the chapter focuses more on how aid workers can be motivated to do well and support the objective of the aid, the idea of justice and injustice is beyond the consideration of most aid workers. In fact, the effectiveness of aid in accelerating development depends on how projects are justified and selected. The selection process is therefore crucial and it must reflect the ideals of fairness and justice, not to mention need. The questions often asked by local communities, politicians and policymakers is how much say they should have in the determination of the type of project or in the level of support that the communities or countries should receive. It is well known that, in some cases, the aid agenda is driven by donor perspective on what is needed in the aid recipient countries. This perspective is all too often devoid of local knowledge and input. Many aid-dependent countries do not have much choice and therefore accept the aid in whatever form they can get it. However, the effectiveness of aid in those circumstances is compromised because the feeling and perception of the aid recipients is that it was not justified in the first place. Of course, there is the well-recorded 'boomerang effect' to consider, when the lion's share of the aid goes back into the pockets of the 
experts and consultants from the donor country, usually in the form of fees and charges.

\section{Identity}

This chapter is an excellent exposition of the behavioural aspect of aid and how it affects the recipients. The key point made by the authors is that donors need to understand the identities of different stakeholders receiving aid before they can feel comfortable that the aid is going to be effective. Usually, aid donors and those assessing aid on the donors' behalf have a dominant position and their identity is paramount in making the final decision. What the chapter on identity brings out is that it is vital that both the donor community and the recipient community understand the relationships with each other and how they can be mutually beneficial. However, it has to be acknowledged that there are aid recipients around the region who continue to manipulate the aid donors to receive aid on a continuous basis without proper analysis and evaluation of its effectiveness. Aid donors have sometimes played to the tune of the powerful aid recipients, especially in the NGO sector, to advance their own special interests. This is often done without actually analysing the real needs on the ground. So the identity of the recipients is crucial and needs to be more prominent. .

\section{Learning}

The chapter on learning provides a fresh perspective on how organisations need to create a learning environment to understand the dynamics of aid implementation. The authors use organisational learning theory to explain the larger issues of national agendas for change. One of the larger issues supported by aid organisations is the economic reform agenda in many developing countries for the last two decades. The economic reforms supported by development partners in many of the Pacific Islands have been only partly successful. This is because the recipient governments and their policymakers found that these reforms were sometimes not incremental and required major changes in culture and attitude. Change requires a lot of organisational learning and, in many instances, this learning did not take place.

\section{Concluding Comments}

The Aid Triangle is an excellent addition to the literature on aid. It provides a different perspective on aid management and the evaluation of its effectiveness. It also raises more questions about the nature of the aid industry and the dynamics involved in it. 